\title{
ANÁLISIS DE ALGUNAS CAUSAS QUE PUEDEN DETERIORAR LOS REVESTIMIENTOS CONTIGUOS CONGLOMERADOS
}

\author{
(ANALYSIS OF SOME OF THE REASONS THROUGH WHICH ADJOINING COVERING \\ CONGLOMERATE COULD BE DAMAGED)
}

Jesús González Martín, Prof. de Construcciones Arquitectónicas de E. U. A.T. M. de la U. P. M.

ESPAÑA

Fecha de recepción: $25-$ VIII-99

\section{RESUMEN}

Se realiza un estudio de los factores que influyen en la degradación de los revestimientos de las edificaciones, haciendo especial hincapié en la relación causalefecto respecto a la problemática de ejecución y restauración de las obras.

\section{SUMMARY}

A study of different factors that could influence the degradation of the building's covering, emphasizing the relationship between causeleffect concerning the difficulties to restaure and execute the works.
Las Leyes de la Naturaleza, mediante acciones físicas, químicas y biológicas, a las que también se ha sumado la contaminación, como componente negativo de la evolución tecnológica y social del hombre, constituyen un compendio de interacciones que modifican nuestro entorno natural y físico.

La escala del tiempo en que actúan estos agentes de intercambio es mayor que la que se desarrolla en torno a nuestra vida cotidiana, por lo que podemos apreciar directamente su velocidad de actuación. Así, elementos que contemplados día a día parecen permanecer inmutables, con el paso de los años descubrimos que han envejecido.

Las edificaciones, no pueden escaparse de las acciones que hemos nombrado anteriormente; si en la Naturaleza podemos aplicar el término transformación, en la edificación deberíamos considerar el de degradación. Esta degradación se produce mayormente en las zonas más expuestas de las fachadas, son diversas y la mayoría motivadas por factores externos, aunque en muchos casos participan cuestiones de diseño, existiendo muchas veces entre ambos una relación.
Estos factores se pueden clasificar en:

\section{Técnicos \\ Físicos \\ Ambientales \\ Sociales \\ Biológicos \\ Mecánicos}

\section{Técnicos}

Una elección equivocada de los materiales ante unas determinadas condiciones ambientales o de uso.

- Un revestimiento adecuado para un clima marino cálido, puede dar un resultado catastrófico en una zona de sierra, con importantes modificaciones térmicas.

- Del mismo modo, un material idóneo en una orientación sur, puede no serlo con una orientación norte y viceversa.

La consideración de que la estructura de un edificio es independiente de los revestimientos, es un error en la 
valoración de la resistencia de los materiales. La incompatibilidad de deformaciones entre la estructura y los cerramientos, así como los asentamientos diferenciales, son factores que pueden producir fisuraciones en los revestimientos de la fachada.

Las formas arquitectónicas evolucionan, pero elproyectista a veces olvida cuestiones que tardaron muchos años en tener un comportamiento óptimo. De este modo, las cornisas podrían responder con garantía de éxito a los ciclos de humedad y secado, pero no sucede lo mismo en una fachada que no tiene cornisas u otro tipo de protecciones similares. Los problemas que comporta esta carencia han de estar compensados con un mejor comportamiento de los revestimientos.

Los errores en la ejecución, pueden ser producidos por diferentes motivos; uno, frecuente, sería que al ser los revestimientos uno de los últimos trabajos que se realizan en una edificación, en muchas ocasiones, se tienen que compensar los gastos de este elemento constructivo con otras partidas agotadas del presupuesto general, por lo que los trabajos no se efectúan con la calidad y garantias exigibles.

\section{Alteraciones fisico-químicas.}

La mayoría de los materiales utilizados pueden resultar afectados por acciones físico-químicas. Consideraremos aquéllas que supongan una agresión destructiva. Su estudio permite actuar en la conservación de los edificios de manera más eficaz.

Los factores de alteración pueden dividirse en dos grupos: internos, aquéllos que afectan a la composición y calidad de los materiales y externos, derivados del uso o de factores ambientales.

Entre los factores internos el más importante es el de la porosidad. Los materiales no porosos suelen deteriorarse en su superficie, mientras que los porosos, aparte de las agresiones superficiales, pueden trasladar, por

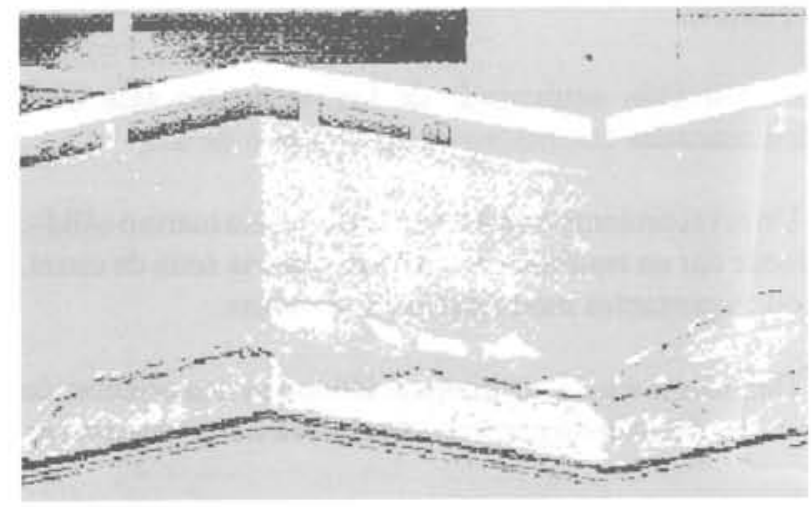

Revoco afectado por diferente comportamiento de dos materiales distintos.

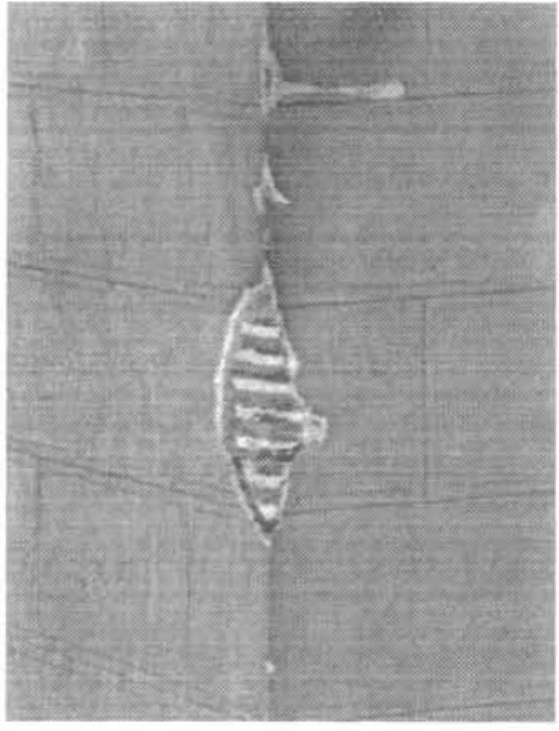

Revoco afectado por acción mecánica.

permeabilidad, los agentes al interior y viceversa; es decir, que los agentes contaminantes y perjudiciales pueden estar contenidos en el mismo material y, con su disolución en agua, ser trasladados al exterior.

\section{Efectos del agua}

El agua es básicamente inestable.

Se puede encontrar en los tres estados, sólido, líquido y gaseoso, bajo condiciones normales de temperatura y presión.

Gran poder como disolvente, pudiendo transportar cualquier partícula que sea soluble en el agua, permitiendo, por tensión superficial, su deslizamiento y penetración en el interior del revestimiento.

\section{Humedad}

La durabilidad de unos revestimientos expuestos al exterior es directamente proporcional a su resistencia a los efectos causados por el agua.

Cuando nos referimos a la humedad, consideramos el agua contenida, más o menos tiempo, en un material poroso.

Normalmente, humedad superficial puede implicar humedad en el interior de la base del revestimiento.

Una superficie seca puede contener un revestimiento húmedo.

Las condiciones climatológicas inciden en el contenido de agua de un material poroso. 
La humedad en un material poroso, puede provenir de diferentes fuentes: por capilaridad en zonas próximas al terreno, por condensación o absorción de la atmósfera o ambiente, por penetración del agua de lluvia o por filtración de instalaciones deterioradas.

\section{Capilaridad}

La existencia de fuerzas capilares asociadas a la tensión superficial, son el fundamento de la ascensión de líquido a través de los capilares.

En un material poroso, el agua se mantiene en equilibrio debido a la acción de tres sistemas de fuerzas: gravitatorias, de capilaridad y de ev qporación, que deben estar relacionadas para que los desplazamientos de agua sean posibles. La aportación de agua se produce por las causas anteriormente reseñadas.

La estructura física de los poros influye de forma determinante en el fenómeno de capilaridad.

\section{- Cerrados:el agua no penetra.}

- Abiertos:el agua penetra más o menos en función de la longitud y sección del capilar, viscosidad del agua, cantidad de aire, etc.

\section{Efectos del viento y agua asociados}

La capa de polvo que se deposita en la superficie de la fachada, combinada con los regueros producidos por el agua, constituyen las manchas en las fachadas con revestimientos continuos. Estas partículas pulverulentas las podríamos clasificar en función del motivo que las produce; pueden ser originadas por la industria, la contaminación ambiental, las partículas minerales en suspensión eólica y por los depósitos de origen vegetal. Estas partículas pulverulentas, independientemente de su procedencia, se adhieren, con más o menos intensidad, al paramento de la fachada, con una atracción gravitatoria dependiente de la configuración arquitectónica del edificio; cuanto menos vertical y rugoso sea el revestimiento, más adherencia se produce. También habría que considerar a) la atracción molecular motivada por la propia naturaleza de las partículas y de la estructura del revestimiento yb) la ocasionada por fuerzas de origen electrostático. Finalmente también tendríamos que considerar la motivada por la adherencia debida a la propia capilaridad y porosidad del paramento.

Como ya hemos indicado anteriormente, la rugosidad del revestimiento influye de modo notorio en el ensuciamiento gradual; existe una relación de proporcionalidad entre la adherencia y la rugosidad; también la dureza del soporte influye cuando las partículas que arrastra el viento inciden sobre el paramento con la energía cinética, pudiendo ser rechazadas o quedarse incrustadas en el revestimiento.

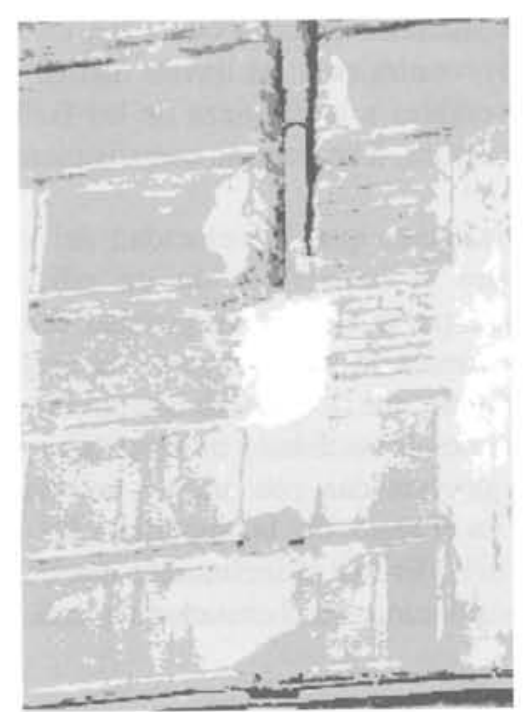

Revoco afectado por rotura de bajante de pluviales.

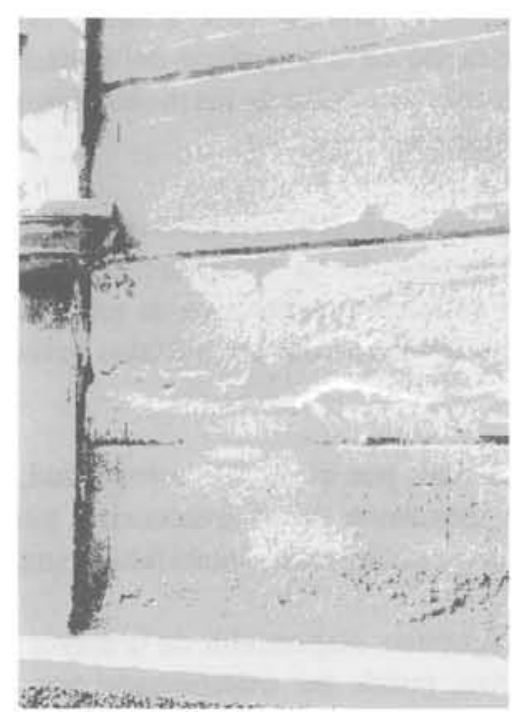

Humedad producida por capilaridad.

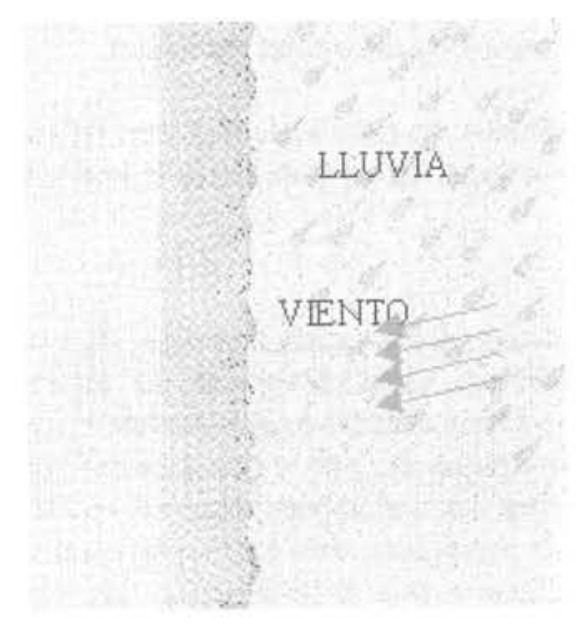

Depósito de suciedad debido a la asociación agualviento.

http://informesdelaconstruccion.revistas.csic.es 
También el agua de lluvia asociada al viento influye en el depósito de suciedad o por el contrario, en la limpieza efectiva del revestimiento: las lluvias fuertes y abundantes, son favorables a la limpieza de las fachadas y las ligeras, por contra, favorecen su ensuciamiento.

Hay que considerar que la velocidad del viento y su acción sobre la superficie de un edificio están condicionadas por la altura, la rugosidad del paramento y la aerodinámica de su configuración arquitectónica. Está demostrado que las gotas de lluvia, contaminadas o no con depósitos de suciedad y transportadas por el fuerte viento, son proyectadas, con mayor intensidad, en las aristas que en el resto de la superficie del mismo. La velocidad del viento y la inclinación que confiera a las gotas de agua, inciden en el ensuciamiento de la fachada.

\section{Eflorescencias}

Son depósitos, en la superficie de los materiales porosos, de sales hidrosolubles. Las sales disueltas en agua, al aflorar en forma de humedad en la fachada y evaporarse, se van concentrando en la superficie del material, con una fuerte tendencia, por parte de las moléculas, a ordenarse para la formación de cristales.

El origen de las sales solubles se determina, generalmente, por los elementos de su composición. Se encuentran asociadas a sulfatos y carbonatos de magnesio, sodio y potasio y, en algunas ocasiones, sulfatos de hierro, cobre, etc.

- Sulfato cálcico: por su escasa solubilidad, interviene poco en la formación de eflorescencias, pero, si se le asocia al sulfato potásico, su solubilidad aumenta.

- Sulfato magnésico: responsable de la mayor parte de las eflorescencias, puede ser transportado por el agua de lluvia solamente.

- Sulfato sódico: su acción destructiva es más severa cuando las sales están en estado de hidratación, pudiendo aumentar cuatro veces su volumen inicial.

- Sulfato férrico: se manifiesta en forma de manchas de orín, debido a que las sales de hierro se han oxidado.

\section{Físicos}

Las alteraciones del revestimiento expuesto a la intemperie, casi siempre, están provocadas por un conjunto de causas que actúan aisladas o conjuntamente. Un aumento de volumen motivado por la cristalización de las sales hidrosolubles, las variaciones térmicas, que se traducen en acciones mecánicas, todo cambio de temperatura que esté asociado a una modificación de volumen, la congelación del agua presente en los capilares del revestimiento, también influyen en el deterioro de la fachada $y$, por supuesto, la abrasión producida por las particulas arrastradas por el viento.

\section{Ambientales}

La emisión de elementos contaminantes lanzados a la atmósfera en forma gaseosa o humos, partículas sólidas en suspensión, condensación de productos sin quemar, etc. constituyen, en todos los casos, elementos perjudiciales para los recubrimientos. El dióxido de carbono por la combustión de motores de automóviles y calefactores, el dióxido de azufre, también originado por la combustión, se puede modificar por efecto de oxidación en $\mathrm{SO}_{3}$ y transformarse en ácido sulfúrico al combinarse con el agua de lluvia.

\section{Sociales}

La sociedad contribuye en gran medida al deterioro urbano, no sólo a través de los conflictos bélicos, sino también por medio de las manifestaciones "informativas o artisticas" que, con pintadas y "graffitis", se muestran intereses seudo-artísticos, protestas, reinvindicaciones u otros tipos de manifestaciones sociales.

En algunos edificios, se manifiesta una deficiencia en la conservación de los elementos comunes, agravado por la creencia de que el propietario de una vivienda lo es también de la parte de fachada del edificio que le corresponde y puede usarla o modificarla a su antojo.

Las edificaciones de uso privado, a diferencia de las públicas, necesitan de un acuerdo común entre los propietarios para poder acometer cualquier obra de acondicionamiento o de simple mantenimiento; esperemos que esto último quede resuelto con la reciente ley municipal de inspección técnica de las viviendas.

También algunas zonas urbanas de carácter residencial se transforman en zonas de servicios y oficinas, produciéndose una ruptura estética entre ambas, por la incorporación de

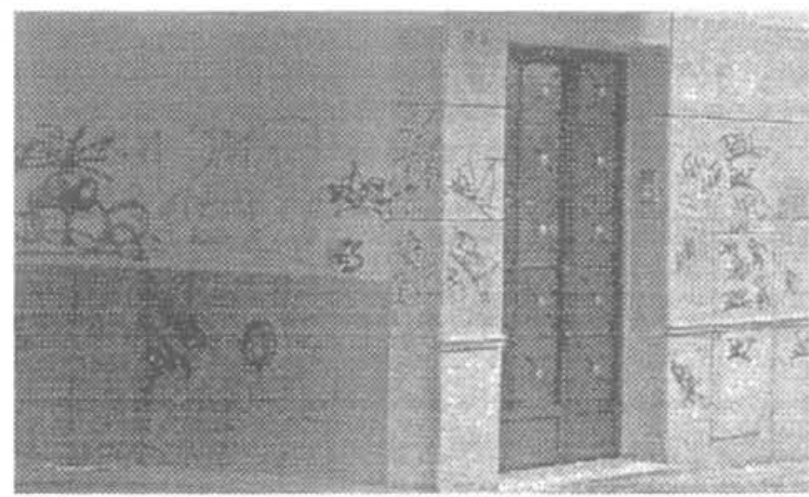

Las pintadas cada vez son más frecuentes en la ciudad. 
otras fachadas, letreros publicitarios, marquesinas, aparatos de aire acondicionado, etc., que afectan a la configuración arquitectónica original.

La permisibilidad de la Administración Local para conseguir las transformaciones parciales en la configuración arquitectónica, con pinturas llamativas, desigual cerramiento de ventanas y terrazas, diferentes tipos y colores en toldos y persianas y un largo muestrario anárquico de formas y colores que se alejan de la necesaria armonía urbana.

Por último, no es menos importante, la desafortunada rehabilitación de algunos edificios que, con aplicación de criterios técnicos erróneos, modifican las características originales sin considerar as ectos, tan importantes en el tiempo de una ciudad, como su uso y la contaminación urbana .

\section{Biológicos}

Producidos por interacción de los seres vivos: como hongos, musgos, líquenes; en fachadas, residuos depositados por animales y microorganismos

\section{Mecánicos}

Sometimiento a tensiones críticas: asentamientos diferenciales, distintos coeficientes de dilatación entre los materiales, cargas excesivas, movimientos del edificio.

\section{Daños más frecuentes en paramentos realizados con revestimientos continuos}

Las fisuras debidas a movimientos estructurales, a deficientes condiciones de aplicación y, en muchos casos,

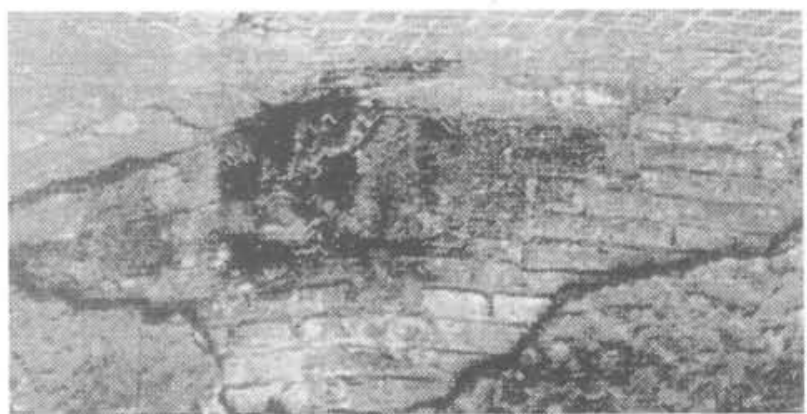

Superficie deteriorada por hongos.

al rigor de la intemperie (contrastes térmicos, heladas, etc.), aceleran el proceso de degradación, con el riesgo de producir, a la larga, desprendimientos parciales del revestimiento.

Otro factor importante de lesiones es la falta de adherencia entre el revestimiento y el soporte, debido a la presencia de sales en la superficie del muro a revestir, degradación del soporte, etc., produciéndose, en consecuencia, grietas, desconchados y desprendimientos del revestimiento.

Por último, no hay que olvidar todos los tipos de ataque físico-químico citados en el apartado anterior.

\section{Cuadros de daños más frecuentes}

Seguidamente se muestran unos resúmenes de cuadros orientativos y generales que establecen una relación entre los síntomas producidos y las posibles causas que los originan. Los primeros, asociados a trabajos generalizados de ejecución o del simple desgaste derivado del uso que lo llamamos "otros" y, los segundos, referidos únicamente a obras de restauración.

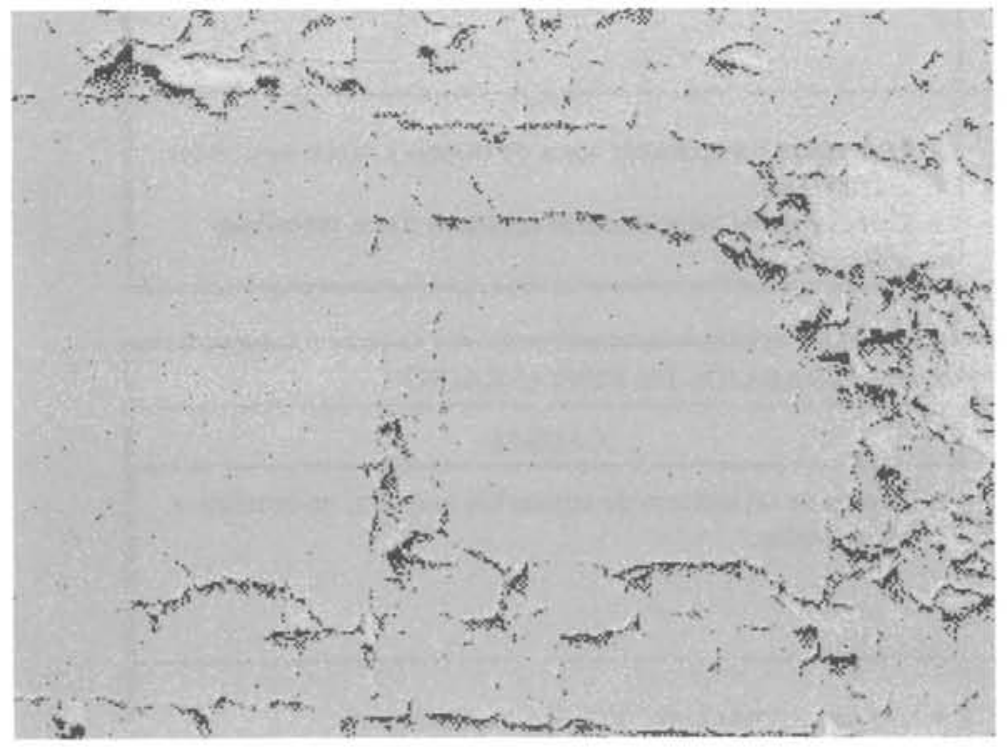

(c) Consejo Superior de Investigaciones Científicas Licencia Creative Commons 3.0 España (by-nc) 


\begin{tabular}{|c|c|}
\hline \multicolumn{2}{|c|}{ PROBLEMAS DEBIDOS A LA EJECUCIÓN Y OTROS } \\
\hline SÍNTOMAS & CAUSAS \\
\hline SUPERFICIE FISURADA & $\begin{array}{l}\text { - Inadecuada proporción de agua aglomerante. } \\
\text { - Secado rápido por prolongada exposición al sol. } \\
\text { - Fraguado demasiado rápido. } \\
\text { - Expansión producida por la adición de yeso en condiciones } \\
\text { de humedad, así como por el ataque de sulfatos. }\end{array}$ \\
\hline $\begin{array}{l}\text { SUPERFICIE DESMORONADA O } \\
\text { DISGREGADA }\end{array}$ & $\begin{array}{l}\text { - Cristalización de sales solubles perjudiciales procedentes de } \\
\text { la base del paramento, componentes sucios y humedades de } \\
\text { capilaridad. } \\
\text { - Soporte base no adecuado o con escasa preparación y/o } \\
\text { adherencia. }\end{array}$ \\
\hline $\begin{array}{l}\text { SEPARACIÓN ENTRE EL } \\
\text { REVESTIMIENTO Y EL SOPORTE, O } \\
\text { SEPARACIÓN ENTRE LAS } \\
\text { DIVERSAS CAPAS, A VECES } \\
\text { ACOMPAÑADO DE SUPERFICIE } \\
\text { AGRIETADA }\end{array}$ & $\begin{array}{l}\text { - Pérdida de adherencia por penetración de agua. } \\
\text { - Acabado del revestimiento con una capa de mayor resistencia } \\
\text { que la capa anterior o la capa base. } \\
\text { - Capas demasído gruesas. } \\
\text { - Aplicación del revestimiento sobre un sopore demasiado } \\
\text { seco. } \\
\text { - Aplicación de un revestimiento impermeable sobre una capa } \\
\text { débil. }\end{array}$ \\
\hline $\begin{array}{l}\text { GRIETAS LONGITUDINALES } \\
\text { ROTURA EN FORMA } \\
\text { LONGITUDINAL U OTRAS FORMAS } \\
\text { ACOMPAÑADAS DE MANCHAS DE } \\
\text { HERRUMBRE. }\end{array}$ & $\begin{array}{l}\text { - Expansión de sulfatos en las juntas, especialmente en los } \\
\text { conductos de salida de humos. } \\
\text { - Corrosión de armaduras. }\end{array}$ \\
\hline $\begin{array}{l}\text { ÁREAS MÁS OSCURAS RODEADAS } \\
\text { POR CÍRCULOS DE } \\
\text { EFLORESCENCIAS PROVOCADAS } \\
\text { POR SALES. }\end{array}$ & - Penetración del agua de lluvia. \\
\hline $\begin{array}{l}\text { SUPERFICIE PARCHEADA DE } \\
\text { MANCHAS DE DISTINTOS } \\
\text { COLORES: NEGRAS, VERDES, } \\
\text { ROSAS, GRISES, BLANCAS. }\end{array}$ & - Depósito de hollín y/o algas, hongos o líquenes. \\
\hline $\begin{array}{l}\text { REVESTIMIENTOS REHINCHADOS } \\
\text { FORMANDO AMPOLLAS, Y } \\
\text { PELÍCULAS BLANDAS PEGAJOSAS } \\
\text { CON REGUEROS AMARILLOS }\end{array}$ & $\begin{array}{l}\text { - Revestimientos aplicados antes de tiempo y sobre superficies } \\
\text { polvorientas. } \\
\text { - Revestimientos oleorresinosos aplicados sobre superficies } \\
\text { húmedas. }\end{array}$ \\
\hline
\end{tabular}

\begin{tabular}{||l|l||}
\hline \multicolumn{2}{|c||}{ PROBLEMAS ASOCIADOS A TRABAJOS DE RESTAURACIÓN } \\
\hline \multicolumn{1}{|c||}{ SÍNTOMAS } & \multicolumn{1}{|c|}{ CAUSAS } \\
\hline $\begin{array}{l}\text { LOS PARCHES EFECTUADOS EN EL } \\
\text { REVOCO APARECEN ROTOS EN SU } \\
\text { PERÍMETRO Y DETERIORO EN SU } \\
\text { ALREDEDOR }\end{array}$ & $\begin{array}{l}\text { Empleo de un mortero de reparación muy rico en cemento e } \\
\text { impermeable. }\end{array}$ \\
\hline & \\
BRILLO SOBRE EL PARAMENTO & - Uso de un componente sintético en el mortero. \\
\hline
\end{tabular}

